\title{
Reduction of Halomethanes with Lithium Aluminum Hydride
}

\author{
By Vernon H. Dibeler
}

\begin{abstract}
A study has been made of the reactions of lithium aluminum hydride with methyl bromide, methylene chloride, methylene bromide, methylene iodide, chloroform, bromoform, and carbon tetrachloride. Except for hydrogen and unreacted halide, essentially pure methane was formed in each case. The amount of hydrogen evolved increased with the number of halogen atoms in the molecule, reaching a maximum in the reduction of carbon tetrachloride. This fact, together with the absence of hydrocarbons heavier than methane, indicates that the reaction proceeds by a step-wise process rather than a simultaneous stripping of several halogen atoms from the molecule.
\end{abstract}

\section{Introduction}

The discovery of lithium aluminum hydride and its effectiveness as a reducing agent [1] ${ }^{1}$ has been followed by its application to the reduction of a wide variety of organic compounds including alkyl and allyl halides, nitriles, aromatic nitro compounds, azoxy compounds, aldimines, epoxides amides, carboxylic acids, aldehydes, ketones, esters, acid chlorides and anhydrides $[2,3,4]$. The reagent has also been used to determine active hydrogen [5]. It does not attack olefinic double bonds; and consequently, has been used to demonstrate the structures of the stereoisomers of 1,3-dichloropropene [6]. It is also reported to reduce anthracene and phenanthrene [7].

In general, reactions that proceed smoothly with lithium aluminum hydride are also those that can be carried out readily with Grignard reagents. An example is the reduction of alkyl halides recently studied by Johnson, Blizzard, and Carhart [3]. These experiments were limited to monohalides with the exception of 1,2-dibromooctane, which in addition to $n$-octane, gave 14 percent unidentified olefin calculated as octene.

Apparent exceptions to the analogy of lithium aluminum hydride and the Grignard reagent have previously been ascribed to differences in the degree of reactivity rather than mode of reaction.

1 Figures in brackets indicate the literature references at the end of this paper.
This paper reports the reduction of a number of halomethanes containing one to four halogen atoms; i. e., methyl bromide, methylene chloride, methylene bromide, methylene iodide, chloroform, bromoform, and carbon tetrachloride. The results show that lithium aluminum hydride is able to react with compounds known to be inert toward the Grignard reagent; viz, the more highly halogenated methanes. Since the experiments were in the nature of a survey, no attempt was made to obtain maximum yields of any of the products.

\section{Experimental Method}

A diagram of the apparatus used in the preparations is given in figure 1. A 250-ml distilling flask $(D)$ was connected to a condenser $(E)$ by a standard tapered joint. The condenser was provided with connections to a vacuum system, an open-end manometer $\left(F^{\prime}\right)$, a trap $(G)$, and a Toepler pump $(H)$. The latter was used to pump reaction products into sample bulbs attached to the standard tapered joint $(I)$. The halides were introduced into the reaction flask at $C$ either by distillation from a break-seal tube $(A)$ or drop-wise from a dropping funnel $(B)$. Standard precautionary techniques used in Grignard reactions were applied throughout. The following description of the reduction of methylene bromide serves to illustrate the method. Approximately $0.5 \mathrm{~g}$ (13 millimoles) 


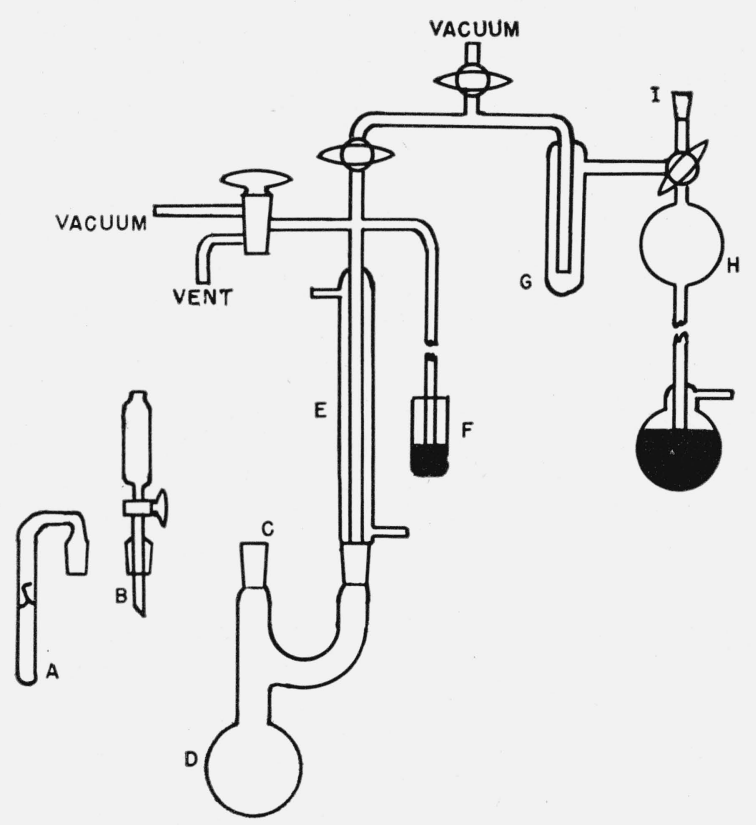

Figure 1. Apparatus for the reduction of halomethanes.

of powdered (under dry nitrogen) lithium aluminum hydride and $50 \mathrm{ml}$ of anhydrous dibutyl ether were introduced into the reaction flask. A break seal tube containing $1.7 \mathrm{~g}$ (10 millimoles) of methylene bromide in vacuum was attached to the flask. The flask was cooled to $0^{\circ} \mathrm{C}$ and cautiously evacuated to degas the mixture. The flask, condenser, and manometer were isolated from the rest of the system and the flask slowly warmed to $60^{\circ} \mathrm{C}$ and held at that temperature for about 3 hrs. Under these conditions gentle refluxing occurred sufficient to stir the mixture. The solution plus some insoluble material was cooled with liquid nitrogen, the flask was thoroughly evacuated and isolated from the pumps. The break-seal tube was opened and the halide condensed onto the frozen ether solution. The flask was allowed to warm slowly until a moderate reaction occurred at about the melting point of the halide. The flask was intermittently cooled to maintain moderate reaction if necessary. When no further rise in pressure was indicated on the manometer, the flask was surrounded with an ice bath at $0^{\circ} \mathrm{C}$. After thermal equilibrium was attained, the pressure of the evolved gases was measured as the difference between the initial and final readings of the manometer. A sample of the product for mass spectrometric analysis was with- drawn by the Toepler pump through the cold trap at $0^{\circ} \mathrm{C}$.

The haloforms and carbon tetrachloride were reduced in a similar manner, except that portions of these compounds were mixed with ether and added drop-wise to the gently refluxing hydride solution at $60^{\circ} \mathrm{C}$.

Dibutyl ether and the halides were laboratorygrade chemicals and were used without purification except for drying. The ether was refluxed for several hours over molten sodium and distilled off. The liquid halides stood for several days over calcium sulfate with occasional shaking. Required samples were removed by pipet through a porous plug. The methyl bromide was not dried.

Mass spectrometric analyses were made with a Consolidated model 21-102 mass spectrometer using conventional procedures [8].

\section{Results}

The experimental results are summarized in table 1. The halomethanes are listed in the first column. Column two gives the nominal temperature at which reduction occurred. Column three gives the ratio of the number of moles of hydride to the number of moles of halide used in the reaction. Column four gives the ratio of the mole percentage of methane to the mole percentage of hydrogen in the evolved gases. Column five gives the percentage yield of methane computed from the halide.

TABLE 1. Summary of experimental results for the reduction. of several halomethanes by lithium aluminum hydride

\begin{tabular}{|c|c|c|c|c|}
\hline Halide & $\begin{array}{l}\text { Nominal tem- } \\
\text { perature of re- } \\
\text { duction }\end{array}$ & $\begin{array}{l}\mathrm{LiAlH}_{4} / \text { halide } \\
\text { molar ratio) }\end{array}$ & $\begin{array}{l}\mathrm{CH}_{4} / \mathrm{H}_{2} \\
\text { (Ratio of } \\
\text { mole \%) }\end{array}$ & Yield \\
\hline & ${ }^{\circ} C$ & & & $\%$ \\
\hline $\mathrm{CH}_{3} \mathrm{Br}$ & Below -80 & 1.0 & 99 & 98 \\
\hline $\mathrm{CH}_{2} \mathrm{Cl}_{2}$ & -80 & 1.1 & 19 & 81 \\
\hline $\mathrm{CH}_{2} \mathrm{Br}_{2}$ & -50 & 1.3 & 13 & 93 \\
\hline $\mathrm{CH}_{2} \mathrm{I}_{2}$ & 10 & 1.0 & 7.3 & 85 \\
\hline $\mathrm{CHCl}_{3}$ & 60 & 1. 6 & 1. 2 & 52 \\
\hline $\mathrm{CHBr}_{3}$ & 60 & 1.5 & 1.1 & 60 \\
\hline $\mathrm{CCl}_{4}$ & 60 & 2. 0 & 0.87 & 36 \\
\hline
\end{tabular}

Except for hydrogen and unreacted halide, mass spectrometric analysis showed that essentially methane was obtained from each halide. The amount of hydrogen produced during the reduction of methyl bromide was less than 1 per- 
cent of the total gas evolved. As shown in table 1 , however, a striking increase in the amount of evolved hydrogen was observed in the reduction of the heavier halides. Some hydrogen was due to water remaining after the halides were dried with calcium sulfate; however, this must be small. It also appears that the over-all yields of methane decrease as the amount of hydrogen increases. The mode of formation of the hydrogen will be considered further in connection with a study with lithium aluminum deuteride.

No hydrocarbons other than methane with appreciable vapor pressures at $0^{\circ} \mathrm{C}$ were observed in any of the products. A small amount of nitrogen retained in the reaction mixture was sometimes evolved with the methane. Since, in the mass spectrum, the most sensitive peak $(m / e=28)$ of both ethylene and ethane coincides with the molecule ion of nitrogen, the low limit of detection of these hydrocarbons was somewhat higher than under jdeal conditions. It is estimated, however, that less than 0.1 percent of either compound was formed in any case. Other hydrocarbons were estimated as less than 0.03 percent.

\section{Discussion}

The prior observations that lithium aluminum hydride can be used to replace the halide of monoalkyl halides with hydrogen has been extended in this paper to show that treatment of halomethanes containing two to four halogen atoms likewise gives methane but in smaller yields. Better yields were obtained with di- and tribromomethane and diiodomethane than with the corresponding chloromethanes. With the monoand dihalogenated methanes, reactions proceeded smoothly at about the melting point of the halide. At room temperature the reaction with the haloforms was slow, and in the case of carbon tetrachloride, little methane was obtained. Accordingly, these reactions were accomplished at higher temperatures.
It is of considerable interest that no hydrocarbons other than methane were detected in any of the reactions. This would indicate that the reduction is a step-wise process, since the simultaneous stripping off of several halogen atoms would greatly increase the probability of coupling to form unsaturated and saturated molecules. It seems equally certain that the reaction does not proceed by a free radical mechanism.

The ability of lithium aluminum hydride to reduce polyhalogenated hydrocarbons as demonstrated above, together with its previously reported effectiveness in reducing monoalkyl halides with out attacking olefinic double bonds, indicate that the hydride offers an attractive method for the preparation of a wide variety of deuterium-substituted compounds, both saturated and unsaturated. Some preliminary work in this laboratory using lithium aluminum deuteride shows that the halomethanes are similarly reduced to give the expected deuterated molecules. However, reactions are somewhat slower and require the utmost in care for high isotopic purity. Further work is in progress and will be described in a later reporit.

\section{References}

[1] A. E. Finholt, A. C. Bond, Jr., and H. I. Schlesinger, J. Am. Chem. Soc. 69, 1199 (1947).

[2] R. F. Nystrom and W. G. Brown, J. Am. Chem. Soc. 69, 1197, 2548 (1947).

[3] J. E. Johnson, R. H. Blizzard, and H. W. Carhart, J. Am. Chem. Soc. 70, 3664 (1948).

[4] R. F. Nystrom and W. G. Brown, J. Am. Chem. Soc. 70, 3738 (1948).

[5] J. A. Krynitsky, J. E. Johnson, and H. W. Carhart, J. Am. Chem. Soc. \%o, 486 (1948).

[6] L. F. Hatch and R. H. Perry, Jr., J. Am. Chem. Soc 71, 3262 (1949).

[7] J. R. Sampey and J. M. Cox, J. Am. Chem. Soc. z1, 1507 (1949).

[8] For a review of recent publications on mass spectrometric techniques, see: J. A. Hipple and M. Shepherd, Anal. Chem. 21, 32 (1949).

Washington, January 9, 1950. 